\title{
Early-Stage Glioblastomas: MR Imaging-Based Classification and Imaging Evidence of Progressive Growth
}

\author{
(D) C.H. Toh and (1) M. Castillo
}

\begin{abstract}
BACKGROUND AND PURPOSE: The serial imaging changes describing the growth of glioblastomas from small to large tumors are seldom reported. Our aim was to classify the imaging patterns of early-stage glioblastomas and to define the order of appearance of different imaging patterns that occur during the growth of small glioblastomas.

MATERIALS AND METHODS: Medical records and preoperative MR imaging studies of patients diagnosed with glioblastoma between 2006 and 2013 were reviewed. Patients were included if their MR imaging studies showed early-stage glioblastomas, defined as small MR imaging lesions detected early in the course of the disease, demonstrating abnormal signal intensity but the absence of classic imaging findings of glioblastoma. Each lesion was reviewed by 2 neuroradiologists independently for location, signal intensity, involvement of GM and/or WM, and contrast-enhancement pattern on MR imaging.

RESULTS: Twenty-six patients with 31 preoperative MR imaging studies met the inclusion criteria. Early-stage glioblastomas were classified into 3 types and were all hyperintense on FLAIR/T2-weighted images. Type I lesions predominantly involved cortical GM ( $n=3$ ). Type II $(n=12)$ and III $(n=16)$ lesions involved both cortical GM and subcortical WM. Focal contrast enhancement was present only in type III lesions at the gray-white junction. Interobserver agreement was excellent ( $\kappa=0.95 ; P<.001$ ) for lesion-type classification. Transformations of lesions from type I to type II and type II to type III were observed on follow-up MR imaging studies. The early-stage glioblastomas of 16 patients were pathologically confirmed after imaging progression to classic glioblastoma.
\end{abstract}

CONCLUSIONS: Cortical lesions may be the earliest MR imaging-detectable abnormality in some human glioblastomas. These cortical tumors may progress to involve WM.

ABBREVIATIONS: $\mathrm{GB}=$ glioblastoma; $I D H I=$ isocitrate dehydrogenase 1

G lioblastoma $(\mathrm{GB})$ is the most common primary malignant brain tumor. It typically appears as a large mass with necrosis, prominent edema, mass effect, and strong heterogeneous contrast enhancement when diagnosed. MR imaging, a noninvasive diagnostic tool with excellent tissue contrast, has the potential to detect small GBs. However, it is uncommon to detect small GBs clinically, probably due to nonspecific or absent symptoms. The serial imaging changes depicting the growth of GBs from small to large tumors are seldom reported.

Received July 25, 2016; accepted after revision September 23

From the Department of Medical Imaging and Intervention (C.H.T.), Chang Gung Memorial Hospital at Linkou and Chang Gung University College of Medicine, TaoYuan, Taiwan; and Department of Radiology (M.C.), University of North Carolina School of Medicine, Chapel Hill, North Carolina.

Please address correspondence to Cheng Hong Toh, MD, PhD, Department of Medical Imaging and Intervention, Chang Gung Memorial Hospital at Linkou, No. 5, Fuxing St, Guishan District, Taoyuan City 333, Taiwan (R.O.C.); e-mail:

eldomtoh@hotmail.com

http://dx.doi.org/10.3174/ajnr.A5015
Some reports described small MR imaging lesions that subsequently progressed to GBs. ${ }^{1-11}$ These are often described as illdefined, FLAIR or T2-weighted hyperintensities without discernable mass effect that typically involve both the cortex and subcortical WM, but occasionally appear as only cortical lesions. ${ }^{2,4,8}$ Contrast enhancement is not a consistent feature and tends to be focal and nodular when present. ${ }^{6-8}$ The commonly affected brain areas include frontal $(n=4),{ }^{2,3,6,8}$ parietal $(n=$ $2),{ }^{7,10}$ occipital $(n=1),{ }^{11}$ temporal $(n=5),{ }^{2,3,6,7,11}$ hippocampal $(n=3),{ }^{1,2,9}$ and insular $(n=1)^{9}$ regions. Because these MR imaging lesions were detected early in the course of the disease, they were frequently referred to as early-stage GBs. ${ }^{3,5-8,11}$

We have noticed different imaging patterns in early-stage GBs. An imaging classification for early-stage GB, however, is not available because most previous studies included only a few such cases. It is important for radiologists to be familiar with early imaging findings and growth patterns of GBs because familiarity may help diagnose small tumors that are symptomatic or incidentally found. Early diagnosis of GB may lead to a higher extent of tumor 
resection, which has been demonstrated to correlate with patient survival. ${ }^{12}$ In this study, we aimed to classify the imaging patterns of early-stage GBs and to the define the order of appearance of different imaging patterns that occur during the growth of these tumors.

\section{MATERIALS AND METHODS}

This retrospective longitudinal observational cohort study was performed in 2 medical centers (Chang Gung Memorial Hospital at Linkou and University of North Carolina Medical Center at Chapel Hill) after institutional review board approval with a waiver of the informed consent requirement. The study was performed in compliance with the Health Insurance Portability and Accountability Act.

A search of the hospital data base was first performed at both institutions for patients diagnosed with glioblastoma between 2006 and 2013. By reviewing their medical records and all available preoperative MR imaging studies, we excluded patients with GBs transformed from histology-proved low-grade gliomas, gliomatosis cerebri, a history of cranial irradiation before the diagnosis of GB, and poor image quality. Patients with preoperative MR imaging studies demonstrating early-stage glioblastomas were included.

Early-stage GBs were defined as small MR imaging lesions detected early in the course of the disease, demonstrating abnormal signal intensity on T2-weighted, FLAIR, and/or postcontrast T1weighted images but with an absence of the imaging findings of classic GB, such as tumors with necrosis, hemorrhage, prominent edema, and heterogeneous contrast enhancement. These lesions were all subsequently proved by histopathology to be GBs. They did or did not develop into classic GBs on MR imaging, depending on the timing of the operation and the frequency of follow-up studies. At each institution, histologic sections were reviewed by neuropathologists with $>15$ years of experience, and diagnosis was made according to World Health Organization criteria.

Besides MR imaging findings, clinical information collected from each patient included age, sex, and tumor isocitrate dehydrogenase (IDH1) gene mutation status determined by immunohistochemistry study, if available.

\section{Imaging Protocols}

Because the MR imaging studies were performed at 2 medical centers with $1.5 \mathrm{~T}$ or $3 \mathrm{~T}$ clinical MR imaging scanners (Magnetom Espree, Avanto, or Tim Trio; Siemens, Erlangen, Germany; Optima MR450w with GEM Suite or Discovery MR750; GE Healthcare, Milwaukee, Wisconsin; or Intera; Philips, Best, the Netherlands), their imaging parameters were not consistent.

The imaging protocol for 1.5T MR imaging scanners typically included transverse T1WI (TR/TE, 449/12 ms; section thickness, $5 \mathrm{~mm}$; gap, $1 \mathrm{~mm}$; matrix, $256 \times 512$; and FOV, $210 \times 178 \mathrm{~mm}$ ), transverse FSE T2WI (TR/TE, 4000/90 ms; section thickness, 5 $\mathrm{mm}$; gap, $1 \mathrm{~mm}$; echo-train length, 17; matrix, $304 \times 512$; and FOV, $210 \times 178 \mathrm{~mm}$ ), transverse FLAIR (TR/TE/TI, 9788/90/ $2300 \mathrm{~ms}$; section thickness, $5 \mathrm{~mm}$; gap, $1 \mathrm{~mm}$; matrix, $256 \times 512$; and FOV, $210 \times 178 \mathrm{~mm})$, and postcontrast T1WI (TR/TE, 420/11 ms; section thickness, $5 \mathrm{~mm}$; gap, $1 \mathrm{~mm}$; matrix, $256 \times$ 512; and FOV , $210 \times 178 \mathrm{~mm}$ ). For $3 \mathrm{~T}$ scanners, the typical pulse sequences included transverse T1WI (TR/TE, 250/2.46 ms; section thickness, $4 \mathrm{~mm}$; gap, $1 \mathrm{~mm}$; matrix, $256 \times 256$; and FOV,
$220 \times 220 \mathrm{~mm}$ ), transverse FSE T2WI (TR/TE, 4000/90 ms; section thickness, $4 \mathrm{~mm}$; gap, 1; flip angle, $120^{\circ}$; echo-train length, 17; matrix, $512 \times 358$; and FOV, $220 \times 220 \mathrm{~mm}$ ), transverse FLAIR (TR/TE/TI, 8200/85/2500 ms; section thickness, $4 \mathrm{~mm}$; gap, $1 \mathrm{~mm}$; matrix, $320 \times 256$; and FOV, $220 \times 220 \mathrm{~mm}$ ), and postcontrast 3D MPRAGE (TR/TE/TI, 2530/4.03/1100 ms; section thickness, $1 \mathrm{~mm}$; matrix, $256 \times 256$; and FOV, $256 \times 224$ $\mathrm{mm}$ ). A standard dose of $0.1 \mathrm{mmol}$ of gadodiamide (Omniscan; GE Healthcare, Piscataway, New Jersey) or gadopentetate dimeglumine (Magnevist; Bayer HealthCare Pharmaceuticals, Wayne, New Jersey) per kilogram of body weight was administered intravenously for postcontrast T1WI or MPRAGE.

\section{Image Analysis}

All available preoperative MR imaging studies for each patient with early-stage GB were reviewed. Two neuroradiologists (C.H.T. and M.C.) with 12 and 26 years of experience, respectively, independently evaluated all lesions for their size, location, involvement of GM and/or WM, MR imaging signal intensity, and contrast-enhancement pattern. Among the imaging features reviewed, involvement of GM and/or WM and contrast-enhancement patterns were used for lesion-type classification. Interobserver differences in lesion type classification were resolved by consensus.

The size of FLAIR/T2-weighted and contrast-enhancing lesions was measured by using a bidimensional method. For each lesion, the largest and perpendicular diameters were measured on a single axial image demonstrating the largest cross-sectional area if the diameters were $>1 \mathrm{~cm}$. Morphologic changes, such as lesion enlargement, new contrast enhancement, or transformation to a classic GB, were recorded when follow-up MR imaging studies were available.

\section{Statistical Analysis}

The level of interobserver agreement for lesion-type classification was determined by calculating the $\kappa$ coefficient. SPSS for Windows, Version 20.0 (IBM, Armonk, New York), was used to perform the statistical analyses, and $P$ values $<.05$ were considered statistically significant.

\section{RESULTS}

Between 2006 and 2013, 282 patients were diagnosed with GBs. Among these, 46 were secondary GBs and 9 were gliomatosis cerebri and therefore were excluded. In the remaining 227 patients, 26 patients (18 from Chang Gung Memorial Hospital at Linkou and 8 from University of North Carolina Medical Center at Chapel Hill) with $31 \mathrm{MR}$ imaging studies met the inclusion criteria. None of these 26 patients had a history of cranial irradiation before the diagnosis of GB or poor image quality. MR imaging findings of early-stage GBs were classified into 3 types on the basis of GM and/or WM involvement and patterns of contrast enhancement. The 2 neuroradiologists agreed on lesion-type classification in 30 of $31 \mathrm{MR}$ imaging studies, with excellent interobserver agreement $(\kappa=0.95 ; P<.001)$. Tables 1,2 , and 3 summarize the clinical characteristics of patients who presented with type I, II, and III lesions, respectively.

Type I lesions were those that on the first available study, pre-

AJNR Am J Neuroradiol 38:288-93 Feb 2017 www.ajnr.org 
Table 1: Clinical characteristics of patients who presented with type I lesions ${ }^{\mathrm{a}}$

\begin{tabular}{|c|c|c|c|c|c|c|c|c|}
\hline \multirow[b]{2}{*}{ Patient } & \multirow[b]{2}{*}{$\begin{array}{c}\text { Sex/Age } \\
\text { (yr) }\end{array}$} & \multirow[b]{2}{*}{ Symptoms } & \multirow[b]{2}{*}{ Lesion Location } & \multirow[b]{2}{*}{$\begin{array}{l}\text { Lesion Size } \\
\text { (cm) }\end{array}$} & \multicolumn{3}{|c|}{ Duration $^{\mathrm{b}}(\mathrm{mo})$} & \multirow[b]{2}{*}{$\begin{array}{c}\text { IDH] } \\
\text { Mutation }\end{array}$} \\
\hline & & & & & $\begin{array}{c}\text { Type } \\
\text { II }\end{array}$ & $\begin{array}{c}\text { Type } \\
\text { III }\end{array}$ & $\begin{array}{c}\text { Classic } \\
\text { GB }\end{array}$ & \\
\hline 1 & $M / 55$ & Focal seizure & Right insula and temporal cortex & 5 & 6 & NA & 11 & - \\
\hline 2 & $M / 57$ & Focal seizure & Left parietal cortex & 2.2 & NA & NA & 7 & + \\
\hline 3 & $M / 60$ & Focal seizure & Right insula and frontal operculum & 3 & NA & NA & 8 & - \\
\hline
\end{tabular}

Note:-NA indicates not available; +, positive; -, negative.

${ }^{a}$ The lesions refer to the FLAIR/T2-weighted hyperintensities predominantly involving cortical GM. Their perpendicular diameters were $<1 \mathrm{~cm}$ and therefore not measured.

${ }^{b}$ The duration is the time interval between the first and follow-up MR imaging studies showing type II lesions, type III lesions, or classic GB, respectively. NA indicates that the particular lesion type was not detected during the course of follow-up.

Table 2: Clinical characteristics of patients who presented with type II lesions ${ }^{\mathrm{a}}$

\begin{tabular}{|c|c|c|c|c|c|c|c|}
\hline \multirow[b]{2}{*}{ Patient } & \multirow[b]{2}{*}{$\begin{array}{c}\text { Sex/Age } \\
\text { (yr) }\end{array}$} & \multirow[b]{2}{*}{ Symptoms } & \multirow[b]{2}{*}{ Lesion Location } & \multirow[b]{2}{*}{$\begin{array}{l}\text { Lesion Size } \\
(\mathrm{cm})\end{array}$} & \multicolumn{2}{|c|}{ Duration $^{\mathrm{b}}$ (mo) } & \multirow[b]{2}{*}{$\begin{array}{c}\text { IDHI } \\
\text { Mutation }\end{array}$} \\
\hline & & & & & $\begin{array}{l}\text { Type } \\
\text { III }\end{array}$ & $\begin{array}{c}\text { Classic } \\
\text { GB }\end{array}$ & \\
\hline 1 & $M / 48$ & LOC & Left temporal & $2 \times 1.2$ & NA & 12 & - \\
\hline 2 & $F / 40$ & Syncope & $\begin{array}{l}\text { Right insula and frontal } \\
\text { operculum }\end{array}$ & $4 \times 2.5$ & NA & 11 & NS \\
\hline 3 & $\mathrm{~F} / 32$ & Speech & Left parietal & $2.5 \times 2$ & NA & 4 & - \\
\hline 4 & $M / 33$ & Headache, LOC & Right insular & $3.1 \times 2.5$ & NA & 4 & NS \\
\hline 5 & $F / 58$ & $\begin{array}{l}\text { Gait disturbance, memory } \\
\text { impairment }\end{array}$ & Right medial frontal & $4 \times 3$ & 10 & 13 & NS \\
\hline 6 & $M / 48$ & Focal seizure & Right parietal & $3.5 \times 2.5$ & 8 & 10 & + \\
\hline 7 & $\mathrm{~F} / 78$ & Headache & Right temporal & $2.5 \times 1$ & NA & 2 & - \\
\hline 8 & $M / 41$ & Focal seizure & Left temporal & $3 \times 2$ & 1 & 1.5 & - \\
\hline 9 & $M / 40$ & Generalized seizure & Left parietal & $3 \times 2.8$ & NA & NA & NS \\
\hline 10 & M/71 & Visual TIA & Right occipital & $3 \times 3$ & 2 & NA & NS \\
\hline 11 & $M / 27$ & Focal seizure & Left frontal & $2.2 \times 2.8$ & NA & NA & + \\
\hline
\end{tabular}

Note:-LOC indicates loss of conscious; NA, not available; NS, not studied; +, positive; -, negative.

${ }^{a}$ The lesions refer to the FLAIR/T2-weighted hyperintensities involving both cortical GM and subcortical WM.

${ }^{b}$ The duration is the time interval between the first and follow-up MR imaging studies showing a type III lesion or classic GB. NA indicates that the particular lesion type was not detected during follow-up. The type II lesions of patients 9 and 11 were resected before they transformed to type III lesions or classic GB.

Table 3: Clinical characteristics of patients who presented with type III lesions ${ }^{\mathrm{a}}$

\begin{tabular}{|c|c|c|c|c|c|c|}
\hline Patient & $\begin{array}{c}\text { Sex/Age } \\
\text { (yr) }\end{array}$ & Symptoms & $\begin{array}{l}\text { Lesion } \\
\text { Location }\end{array}$ & $\begin{array}{l}\text { Lesion Size } \\
(\mathrm{cm})\end{array}$ & $\begin{array}{l}\text { Duration }{ }^{\mathrm{b}} \text { (mo) } \\
\text { to Classic GB }\end{array}$ & $\begin{array}{c}\text { IDHI } \\
\text { Mutation }\end{array}$ \\
\hline 1 & $F / 95$ & Facial palsy & Right frontal & $3 \times 1.5$ & 3 & NS \\
\hline 2 & $M / 48$ & Generalized seizure & Right frontal & $4 \times 2$ & NA & - \\
\hline 3 & $M / 69$ & Focal seizure & Right parietal & $2.5 \times 1.2$ & 3 & - \\
\hline 4 & $\mathrm{~F} / 59$ & Slurred speech & Left insular & $3 \times 1.3$ & NA & NS \\
\hline 5 & $\mathrm{~F} / 55$ & Hand numbness & Right parietal & $2.1 \times 1.3$ & NA & - \\
\hline 6 & $F / 69$ & Leg weakness & Right frontal & $3 \times 1.2$ & NA & NS \\
\hline 7 & $\mathrm{~F} / 35$ & Leg numbness & Left frontal & $2.5 \times 1.3$ & 1 & - \\
\hline 8 & $M / 65$ & Generalized seizure & Left parietal & $2.6 \times 2.6$ & NA & NS \\
\hline 9 & $M / 45$ & Generalized seizure & Left temporal & $3 \times 3$ & 1 & NS \\
\hline 10 & $M / 58$ & Generalized seizure & Left frontal & $1.5 \times 1$ & NA & NS \\
\hline 11 & $M / 43$ & Generalized seizure & Left frontal & $3 \times 1.8$ & NA & NS \\
\hline 12 & $M / 62$ & Generalized seizure & Left temporal & $2.4 \times 2.3$ & 6 & - \\
\hline
\end{tabular}

Note:-NA indicates not available; NS, not studied; -, negative.

${ }^{a}$ The lesions refer to FLAIR/T2-weighted hyperintensities involving the cortex and subcortical WM, with focal contrast enhancement at the GM-WM junction. The size of contrast-enhancing foci is not shown because they are about $\leq 1 \mathrm{~cm}$.

${ }^{b}$ The duration is the time interval between the first and follow-up MR imaging studies showing classic GB. NA indicates that type III lesions were resected before progressing to classic GB.

dominantly involved GM (ie, the cerebral cortex). They appeared as T2-weighted or FLAIR hyperintensities without contrast enhancement. Three patients had type I lesions (Fig 1). Type II lesions were T2-weighted or FLAIR hyperintensities involving the cortex and subcortical WM without contrast enhancement. Twelve patients, including one who progressed from a type I lesion, had type II lesions (Fig 2). Type III lesions were hyperintense on T2-weighted and FLAIR images, involved both the cortex and subcortical WM, and demonstrated small focal areas of contrast enhancement at the GM-WM junction. The diameter of enhancing focus was about $\leq 1 \mathrm{~cm}$ in all. Sixteen patients, including 4 who progressed from type II, had type III lesions (Fig 3).

Figure 4 summarizes the morphologic changes of the earlystage GBs in 26 patients as observed in follow-up MR imaging studies. One type I lesion transformed to a type II, and 4 type II lesions transformed to type III. Reverse-order transformation (ie, transformation from a type III lesion to types II and I or from type II to I) was not observed.

Two type II and 8 type III lesions underwent an early operation 

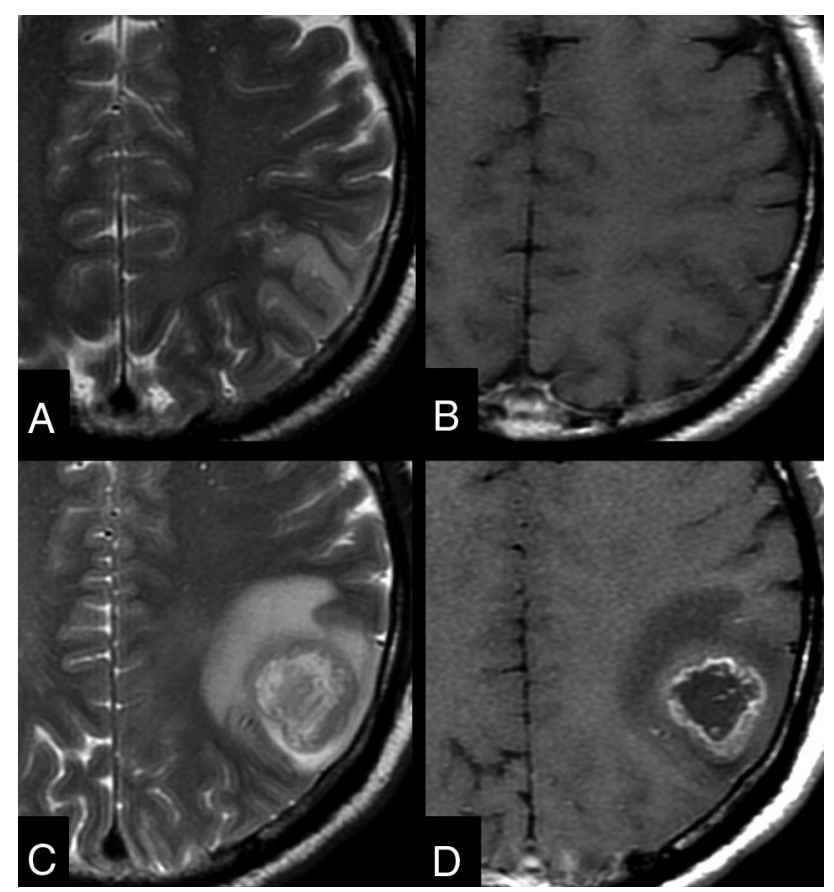

FIG 1. A 57-year-old man with a type I lesion. Axial images show a left parietal cortical lesion, which is hyperintense on the T2-weighted image $(A)$ and without enhancement on the contrast-enhanced T1weighted image $(B)$. T2-weighted $(C)$ and contrast-enhanced T1weighted $(D)$ images obtained 7 months later show a left parietal glioblastoma.
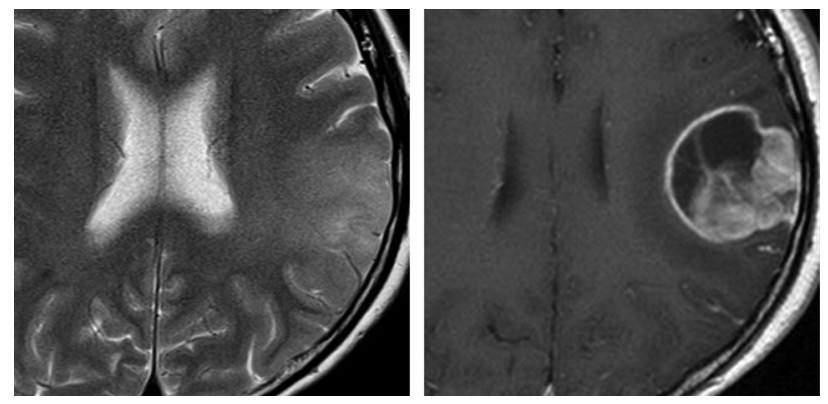

FIG 2. Axial images in a 32-year-old woman with a type II lesion. Left, T2-weighted image shows hyperintensity involving the left parietal cortex and subcortical WM. Right, contrast-enhanced T1-weighted image obtained 4 months later shows a left parietal glioblastoma.
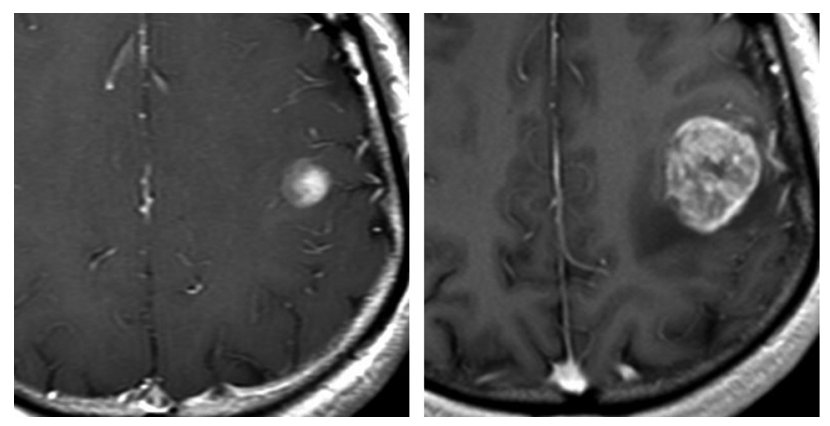

FIG 3. Contrast-enhanced T1-weigthed axial images in a 35-year-old woman with a type III lesion. Left, a cortical/subcortical contrastenhancing lesion is present in the left frontal region. Right, the lesion transformed to a left frontal glioblastoma after 1 month.

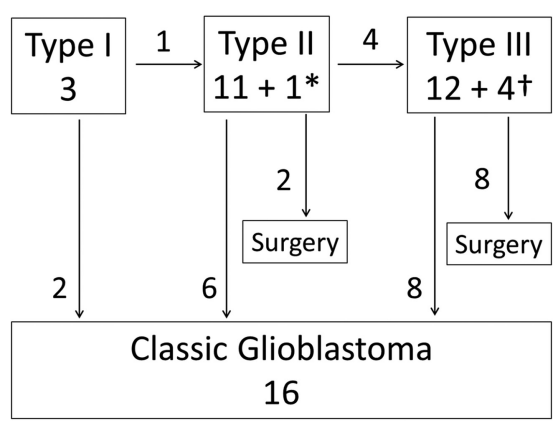

FIG 4. Diagram summarizes the morphologic changes of the 26 earlystage glioblastomas observed in follow-up MR imaging studies. The asterisk and dagger indicate the number of lesions transformed from type I and type II lesions, respectively.

and were confirmed to be GBs. The early-stage GBs of 16 patients were surgically resected after imaging progression to classic GB was documented. IDH1 mutation status was available in 14 GBs, with 3 being positive for this mutation. The IDH1 mutation rate was $21.4 \%$ ( 3 of 14 ).

\section{DISCUSSION}

In this study, we propose an MR imaging-based classification for early-stage GBs. Lesions were classified into 3 types on the basis of the involvement of GM and/or WM and their patterns of contrast enhancement. The 3 types of MR imaging lesions may represent sequential stages of human GB growth. To the best of our knowledge, an MR imaging-based classification for early-stage GBs does not exist.

Among the 3 types of lesions, type I was the earliest, followed by type II, and then type III, according to the morphologic changes observed on follow-up MR imaging studies. The order of appearance suggests that some GBs start as T2-weighted or FLAIR hyperintense lesions in the GM (ie, cerebral cortex [type I]). Then, both the cortex and subcortical WM become involved (type II). Later, focal contrast enhancement develops at the GM-WM junction, within the area of T2-weighted or FLAIR hyperintensity (type III). Enlargement of the contrast-enhancing focus then evolves into the classic appearance of GB.

Our review of the literature shows that there were 19 cases of early-stage GBs with MR imaging studies included when they were published. ${ }^{1-11}$ With our proposed classification, 2 of 19 lesions may be classified as type $\mathrm{I}^{2,8} ; 12$, as type $\mathrm{II}^{1-3,8-11}$; and 5 , as type III. ${ }^{4,6-8}$ Oyama et $\mathrm{al}^{8}$ emphasized the role of DWI in early tumor detection when they reported a GB that first appeared as a type I lesion, then transformed to a type II lesion, and finally transformed to a classic GB before the operation. A type I lesion reported by Thaler et $\mathrm{al}^{2}$ was described as a "right medial frontal nondiagnostic T2-weighted hyperintensity."

It is difficult to study the cell of origin and growth of human GB because the tumors are typically large and in their late stage when diagnosed. Therefore, genetically engineered mouse models in which gliomas are induced by manipulation of the mouse genome at the molecular level are important tools for studying gliomagenesis. ${ }^{13,14}$ Using mosaic analysis with a double marker genetic mouse model, Liu et a ${ }^{15}$ discovered that an oligodendrocyte precursor cell was the cell of origin of malignant gliomas and 
that the earliest neoplastic lesions were found in the GM. Furthermore, they observed tumor extension in subcortical regions along WM tracts. ${ }^{15}$ Another study also found that a glioblastoma could originate from cortical neurons. ${ }^{16}$ However, there is always concern about whether results from animal studies can be transferred to humans. It is not known whether gliomas growing in mice with genetic alterations and different microenvironments resemble spontaneous human GBs.

In our present study, we found that GM lesions were the earliest MR imaging-detectable abnormalities during human GB growth. We believe this finding may serve as indirect evidence, along with that found in the mouse glioma models, ${ }^{15,16}$ to suggest that some GBs may originate from GM. Moreover, the WM FLAIR/T2-weighted hyperintensity of type II lesions may correspond to GB infiltration rather than just edema. GBs have also been reported to originate and recur in the subventricular zone, and it is possible that tumors arising in the cortex are due to secondary outward migration of abnormal brain tumor cells. ${ }^{17,18}$ These issues are beyond the scope of this article, and we wish to only describe and emphasize a subset of GBs that originate in the cortex and are probably from a different cell origin than periventricular or deep WM GBs.

In the present study, the diagnosis of GB in 16 patients was confirmed after their MR imaging lesions progressed to classic ones. Thus, it is possible that those 16 patients initially had lowgrade gliomas, which later transformed to secondary GBs. IDH1 mutation status has recently been considered a molecular marker of secondary GBs. The reported IDH1 mutation rates for clinically diagnosed primary and secondary GBs are about $4 \%-7 \%$ and $73 \%-88 \%$, respectively. ${ }^{19}$ Among those 16 patients, $I D H 1$ mutation status was available in 11 with 2 being positive. The mutation rate of our patients is lower than expected for secondary GBs. As reported in previous studies, the median intervals for low-grade gliomas to transform to GBs ranged from 2.1 to 10.1 years. ${ }^{20}$ The MR imaging lesions of those 16 patients progressed to the classic appearance of GBs in $<14$ months. Among these, the 2 lesions with IDH1 mutations progressed to classic GB in 7 and 10 months, respectively. In light of rapid progression to GBs and the low incidence of IDH1 mutation, we believe the MR imaging lesions of those 16 patients were not low-grade gliomas but highgrade from their origin.

The differential diagnosis for type I lesions should include postictal change because focal seizures were the clinical presentation for 3 patients with type I lesions. However, in previous reports, most seizure-induced MR imaging abnormalities were transient and reversible. ${ }^{21}$ Permanent structural abnormalities such as gliosis and focal atrophy are more likely to occur in status epilepticus. ${ }^{21}$ In this study, no patients with type I lesions had status epilepticus, and their abnormalities in the cerebral cortex persisted even when these lesions transformed to type II or classic GBs. Moreover, seizure-induced abnormalities tend to involve both the cortex and subcortical WM and are seldom limited to GM only. ${ }^{21}$ Therefore, our type I lesions likely reflect tumor in GM.

According to the histologic classification of the World Health Organization, the presence of microvascular proliferation or pseudopalisading necrosis differentiates GBs from lower-grade gliomas. These 2 histologic hallmarks are typically present in the contrast-enhancing component of GB. ${ }^{22,23}$ Microvascular proliferation is known to result in neovascularity with a disrupted blood-brain barrier and increased permeability to gadoliniumbased contrast agents and thus contrast enhancement in GB. Barajas et $\mathrm{al}^{22}$ reported that tissue samples obtained from nonenhancing tumor components of GBs demonstrated less microvascular proliferation than those from contrast-enhancing components. GBs without contrast enhancement, though relatively rare, have been reported. ${ }^{23-25}$ In the study of Utsuki et al, ${ }^{23}$ purely non-contrast-enhancing glioblastomas demonstrated only pseudopalisading necrosis and no neovascularity. Although histopathologic diagnosis was only available in 2 type II lesions, we believe that the other 10 type II lesions were also GBs without contrast enhancement. The implication of these observations may be that advanced imaging techniques such as perfusion and permeability may not reflect the true nature of these small GBs as they do in larger, classic ones.

The present study helps radiologists be familiar with imaging findings of early-stage GBs. For a type I or II lesion, early-stage GB should be included in the differential diagnosis in addition to a self-limited non-neoplastic lesion. It is challenging to prospectively diagnose early-stage GBs; therefore, aggressive surgical resection of these lesions is unlikely. Biopsy may be an alternative other than short-interval imaging follow-up. Advanced MR imaging such as diffusion, perfusion, and MR spectroscopy may have a role, but further studies are needed.

There are some limitations to our study. First, the diagnosis of GB was confirmed in only 10 patients who underwent an early operation. Thus, for the other 16 patients, we can only assume that their MR imaging lesions were early-stage GBs because they all eventually developed the typical MR imaging appearance and histopathology of GBs. Second, because our routine MR imaging protocol did not include advanced MR imaging techniques such as diffusion, perfusion, and spectroscopy, we could not assess their roles in early-stage GBs. Third, the MR imaging findings describe only macroscopic growth of GBs and not their microscopic changes. Although GBs may arise from GM, their cell of origin remains unknown. Fourth, tumors included in this study probably represent only a subset of GBs. GBs arising from the hippocampus and subventricular zone may have different growth patterns on MR imaging. Finally, due to a limited number of patients and the retrospective nature of our study, we were unable to determine whether our MR imaging-based classification correlates with outcomes.

\section{CONCLUSIONS}

A cortical GM lesion may be the earliest MR imaging-detectable abnormality in some human GBs. GBs may originate from the cortical GM and extend into the subcortical WM. Detection of these lesions while limited to the GM may allow total resection and potentially improve patient outcome.

\section{REFERENCES}

1. Nam TS, Choi KH, Kim MK, et al. Glioblastoma mimicking herpes simplex encephalitis. J Korean Neurosurg Soc 2011;50:119-22 CrossRef Medline 
2. Thaler PB, Li JY, Isakov Y, et al. Normal or non-diagnostic neuroimaging studies prior to the detection of malignant primary brain tumors. J Clin Neurosci 2012;19:4111-14 CrossRef Medline

3. Ideguchi M, Kajiwara K, Goto H, et al. MRI findings and pathological features in early-stage glioblastoma. J Neurooncol 2015;123: 289-97 CrossRef Medline

4. Takagi I, Shakur SF, Lukas RV, et al. Spontaneous radiographic resolution and subsequent redemonstration of an untreated glioblastoma. J Neurosurg 2011;115:24-29 CrossRef Medline

5. Nishi N, Kawai S, Yonezawa T, et al. Early appearance of high grade glioma on magnetic resonance imaging. Neurol Med Chir (Tokyo) 2009;49:8-12 CrossRef Medline

6. Simonet Redondo M, Auger Acosta C, Rovira-Gols A, et al. Neuroimaging findings in the initial phase of development of high grade cortical astrocytomas. Radiologia 2012;54:424-31 CrossRef Medline

7. Rossi R, Figus A, Corraine S. Early presentation of de novo high grade glioma with epileptic seizures: electroclinical and neuroimaging findings. Seizure 2010;19:470-74 CrossRef Medline

8. Oyama H, Ando Y, Aoki S, et al. Glioblastoma detected at the initial stage in its developmental: case report. Neurol Med Chir (Tokyo) 2010;50:414-17 CrossRef Medline

9. Cohen-Gadol AA, DiLuna ML, Bannykh SI, et al. Non-enhancing de novo glioblastoma: report of two cases. Neurosurg Rev 2004;27: 281-85 Medline

10. Okamoto K, Ito J, Takahashi N, et al. MRI of high-grade astrocytic tumors: early appearance and evolution. Neuroradiology 2002;44: 395-402 CrossRef Medline

11. Jung TY, Jung S. Early neuroimaging findings of glioblastoma mimicking non-neoplastic cerebral lesion. Neurol Med Chir (Tokyo) 2007;47:424-27 CrossRef Medline

12. Marko NF, Weil RJ, Schroeder JL, et al. Extent of resection of glioblastoma revisited: personalized survival modeling facilitates more accurate survival prediction and supports a maximum-safe-resection approach to surgery. J Clin Oncol 2014;32:774-82 CrossRef Medline

13. Chen J, McKay RM, Parada LF. Malignant glioma: lessons from genomics, mouse models, and stem cells. Cell 2012;149:36-47 CrossRef Medline

14. Jiang Y, Uhrbom L. On the origin of glioma. Ups J Med Sci 2012;117: 113-21 CrossRef Medline

15. Liu C, Sage JC, Miller MR, et al. Mosaic analysis with double markers reveals tumor cell of origin in glioma. Cell 2011;146:209-21 CrossRef Medline

16. Friedmann-Morvinski D, Bushong EA, Ke E, et al. Dedifferentiation of neurons and astrocytes by oncogenes can induce gliomas in mice. Science 2012;338:1080-84 CrossRef Medline

17. Das S, Srikanth M, Kessler JA. Cancer stem cells and glioma. Nat Clin Pract Neurol 2008;4:427-35 CrossRef Medline

18. Achanta P, Sedora Roman NI, Quiñones-Hinojosa A. Gliomagenesis and the use of neural stem cells in brain tumor treatment. Anticancer Agents Med Chem 2010;10:121-30 CrossRef Medline

19. Ohgaki $H$, Kleihues $P$. The definition of primary and secondary glioblastoma. Clin Cancer Res 2013;19:764-72 CrossRef Medline

20. Sanai N, Chang S, Berger MS. Low-grade gliomas in adults. J Neurosurg 2011;115:948-65 CrossRef Medline

21. Cianfoni A, Caulo M, Cerase A, et al. Seizure-induced brain lesions: a wide spectrum of variably reversible MRI abnormalities. Eur J Radiol 2013;82:1964-72 CrossRef Medline

22. Barajas RF Jr, Phillips JJ, Parvataneni R, et al. Regional variation in histopathologic features of tumor specimens from treatment-naive glioblastoma correlates with anatomic and physiologic MR imaging. Neuro Oncol 2012;14:942-54 CrossRef Medline

23. Utsuki S, Oka H, Miyajima Y, et al. Glioblastoma without remarkable contrast enhancement on magnetic resonance imaging. Int J Clin Med 2012;3:439-45 CrossRef

24. Pope WB, Sayre J, Perlina A, et al. MR imaging correlates of survival in patients with high-grade gliomas. AJNR Am J Neuroradiol 2005; 26:2466-74 Medline

25. Knopp EA, Cha S, Johnson G, et al. Glial neoplasms: dynamic contrast-enhanced T2*-weighted MR imaging. Radiology 1999;211: 791-98 CrossRef Medline 